\title{
Light curve solutions of the ultrashort-period Kepler binaries *
}

\author{
D. Kjurkchieva ${ }^{1, \star \star}$ and D. Dimitrov ${ }^{2}$ \\ 1 Department of Physics, Shumen University, 115 Universitetska, 9700 Shumen, Bulgaria \\ 2 Institute of Astronomy and NAO, Bulgarian Academy of Sciences, Tsarigradsko shossee 72, 1784 Sofia, Bulgaria
}

Received ... 2014, accepted ... 2014

Published online later

Key words binaries: close - binaries: eclipsing - methods: data analysis - stars: fundamental parameters - stars: individual (KID 4921906, KID 1572353, KID 8108785, KID 6309193, KID 12055255, KID 9532219)

\begin{abstract}
We carried out light curve solutions of the ultrashort-period binaries with MS components observed by Kepler. All six targets turned out almost in thermal contact with contact or slightly overcontact configurations. Two of them, KID 4921906 and KID 6309193, are not eclipsing but reveal ellipsoidal and spot variability. One of the components of KID 8108785 exhibits inherent, quasi-sinusoidal, small-amplitude variability. KID 12055255 turned out a very rare case of ultrashortperiod overcontact binary consisting of two M dwarfs. Our modeling indicated that the variability of KID 9532219 is due to eclipses but not to $\delta$ Sct pulsations as it was previously supposed.
\end{abstract}

(C) 0000 WILEY-VCH Verlag GmbH \& Co. KGaA, Weinheim

\section{Introduction}

Most of the contact binaries consisting of solar-type components have orbital periods within $0.25 \mathrm{~d}<P<0.7 \mathrm{~d}$. Rucinski (1992) found that they show a short-period limit at about $0.22 \mathrm{~d}$. But the modern large surveys allowed to discover targets beyond this limit (Maceroni \& Rucinski 1997; Maceroni \& Montalban 2004; Weldrake 2004; Rucinski 2006, 2007; Pribulla et al. 2009; Lohr et al. 2014; Qian et al. 2014, etc.).

The well-studied binaries with periods around and below cut-off limit (ultrashort-period systems) and MS components are quite rare but important objects for the astrophysics, especially for the stellar evolution.

Until two decades ago the statistics of contact binaries was particularly affected by the trend to discover relatively large light-curve amplitudes, but very recently, the huge surveys (ROTSE, MACHO, ASAS, SuperWASP, etc.) led to discoveries of many low-amplitude systems.

Lately, Kepler provides unique, unprecedented precise, extended and nearly uninterrupted data set for a large number and variety of stars. Above two thousands eclipsing binaries (EBs) were identified and included in the Kepler EB catalog (Prsa et al. 2011, further PRSA11; Slawson et al. 2011). According to the initial classification the sample consists of 1261 detached, 152 semidetached, 469 overcontact binaries, 137 ellipsoidal variables (they are not EBs!), and 146 uncertain systems. The automated fitting of the light curves by a polynomial chain and the artificial intelligence based tier EBAI (Prsa et al. 2008) were used for the morpho-

\footnotetext{
* Data from Kepler

$\star \star$ Corresponding author: e-mail: d.kyurkchieva@shu-bg.net
}

logical classification and determination of the global parameters of some systems of the Kepler EB catalog.

Several individual Kepler EBs were studied in details (e.g., Southworth et al. 2011; Steffen et al. 2011; Welsh et al. 2011; Winn et al. 2011). This paper is devoted to standard ("manual") light curve solutions of the ultrashort-period binaries from the Kepler EB catalog.

\section{Selection of targets and preliminary preparation of the data}

As a continuation of our interest in binaries with extremely small orbital periods (Dimitrov \& Kjurkchieva 2010), we reviewed the Kepler EB catalog. It contains 22 targets with periods $P<0.23 \mathrm{~d}$ (for better statistics we put an upper limit $0.23 \mathrm{~d}$ that is slightly bigger than the cut-off limit of $0.22 \mathrm{~d}$ ).

Although Prsa et al. (2011) reported about visual inspection of every target and culling about $27 \%$ of their initial sample as nonEBs (RR Lyr-, $\gamma$ Dor-, $\delta$-Sct type stars), we carried out new visual review of the chosen 22 targets. We concluded that 13 ultrashort-period stars are $\delta$ Sct variables due to the shape of their curves (KID 10417986, KID 8912468, KID 8758716, KID 10855535, KID 9612468, KID 8912730, KID 9898401, KID 7375612, KID 5872696, KID 10684673, KID 6699679, KID 6287172, KID 11825204). Our classification of these stars is supported by their high temperatures (above $6000 \mathrm{~K}$ ).

One ultrashort-period star, KID 9472174, turned out to be the known sdB+dM binary $2 \mathrm{M} 1938+4603$.

The shapes of the light curves of two ultrashort-period targets, KID 8288741 and KID 12350008, seem as those of detached eclipsing systems (with almost flat maxima) but 
their amplitudes of 1-2 mmag and periods are absolutely inappropriate for such configurations. Our preliminary attempts to reproduce their data required enormous big third light. This means that KID 8288741 and KID 12350008 are not the true variable stars. It is meaningless to model these data because the obtained parameters would not be reliable. Matuevic et al. (2012) noted that the automated classification and modeling of Kepler EBs, even with locally linear embedding, failed for small amplitudes ( $\sim 0.001 \mathrm{mag})$. KID 8288741 and KID 12350008 are such cases but the reason to be removed from the further analysis is the discrepancy between the light curve shape appropriate for detached eclipsing binary and the negligible amplitude of variability (smaller even than those of ellipsoidal variations).

As a result of the selection procedure we gathered sample of six ultrashort-period binaries: KID 4921906, KID 1572353, KID 8108785, KID 6309193, KID 12055255, KID 9532219. More conclusive confirmation of their duplicity would be spectroscopic data. The objects would require $8 \mathrm{~m}$-class telescope due to low brightness and short period at the same time. Another possibility for proper classification is the color-period diagram (Duerbeck, 1997) but the presence of third component or close neighbor can alter the color information. So we will use the important criterion for sure classification of the targets: the successful reproducing of their light curves by effects of duplicity.

Table 1 presents information for our 6 targets from the Kepler EB catalog: Kepler magnitude $m_{K}$; epoch $T_{0}$ of the primary minimum; orbital period $P$; mean temperature $T_{m}$; metallicity $[\mathrm{Fe} / \mathrm{H}]$; radius $R$; $\log g$; reddening $\mathrm{E}(\mathrm{B}-\mathrm{V})$; type of configuration; ratio $T_{2} / T_{1}$ of the temperatures of the components; fillout factor $F F$; crowding (a number between 0 and 1 that specifies the fraction of flux in the optimal photometric aperture due to the target star with respect to the total flux from all sources).

More than 50000 points are available for each target in the Kepler archive. The detailed review of these data revealed that the shape of the light curves almost did not change during the different observational quarters. However the levels of maxima and minima and the amplitudes of the light curves of the most targets changed considerably during the different quarters causing the big thickness of the total light curves (from all quarters). This effect is probably due to problems of the satellite steering and automated reduction of the data. That is why we assumed that it is reasonable to model data from several consecutive quarters rather than all data simultaneously. Moreover, we established that it is more appropriate to use the raw Kepler data and to make own reduction and de-trending (Dimitrov et al. 2012).

We chose to model the data from quarters Q0 and Q1 for all targets excepting KID 9532219 which observations began later. Their folded light curves are presented in Figs. $1-6$.

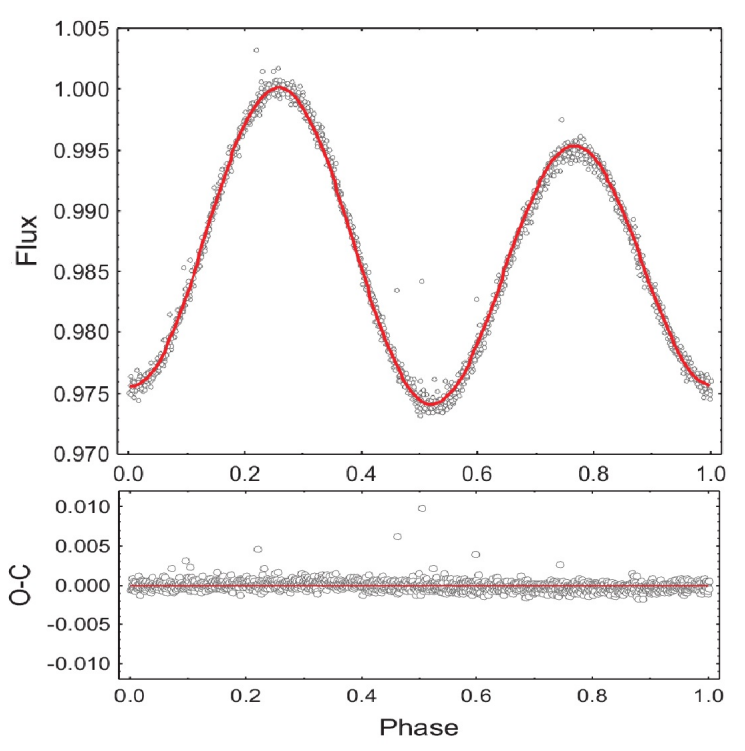

Fig. 1 The light curve of KID 4921906 and our fit (top) and the corresponding residuals (bottom)
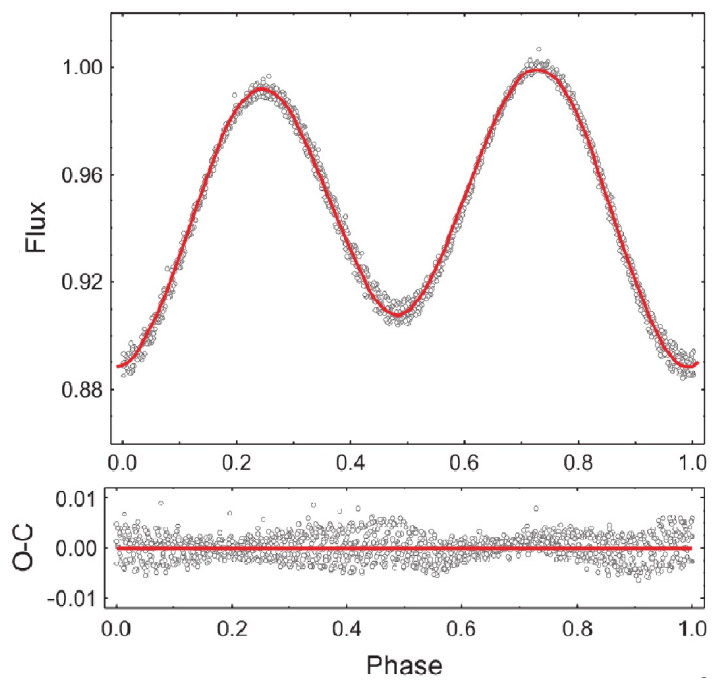

Fig. 2 The light curve of KID 1572353 and our fit (top) and the corresponding residuals (bottom)

\section{Light curve solutions}

The shapes of the light curves (Figs. 1-6) implied that our targets are nearly contact or overcontact systems that was expected for their short orbital periods. The depths of the two minima of the most curves are close that means that the binaries are almost in thermal contact. The small light amplitudes of some targets point at low orbital inclinations and consequently, considerable contributions of the ellipsoidal variations.

Taking into account the foregoing considerations we carried out modeling of the photometric data by the code PHOEBE (Prsa \& Zwitter 2005) using the following procedure. 
Table 1 Parameters of the targets from the EB catalog

\begin{tabular}{ccccccccccccc}
\hline Kepler ID & $\begin{array}{c}m_{K} \\
{[\mathrm{mag}]}\end{array}$ & $\begin{array}{c}T_{0}-2400000 \\
\text { [days] }\end{array}$ & $\begin{array}{c}P \\
\text { [days] }\end{array}$ & $\begin{array}{c}T_{m} \\
{[\mathrm{~K}]}\end{array}$ & {$[\mathrm{Fe} / \mathrm{H}]$} & $\begin{array}{c}R \\
{\left[R_{\odot}\right]}\end{array}$ & $\log g$ & $\mathrm{E}(\mathrm{B}-\mathrm{V})$ & Type & $T_{2} / T_{1}$ & $F F$ & crowd \\
\hline 4921906 & 15.203 & 54965.148704 & 0.213732 & 4800 & -0.394 & 0.99 & 4.41 & 0.109 & $\mathrm{OC}$ & 0.958 & 1.033 & 0.648 \\
1572353 & 15.234 & 55001.306290 & 0.228900 & 4470 & -0.261 & 0.77 & 4.54 & 0.076 & $\mathrm{OC}$ & 0.883 & 1.051 & 0.9 \\
8108785 & 14.757 & 54964.641700 & 0.228840 & 4354 & -0.298 & 0.67 & 4.61 & 0.053 & OC & 0.855 & 0.414 & 0.921 \\
6309193 & 13.674 & 55002.434080 & 0.212500 & 5367 & -0.383 & 1.09 & 4.38 & 0.098 & OC & 1.012 & 1.01 & 0.92 \\
12055255 & 15.866 & 54964.966610 & 0.220950 & 4093 & 0.106 & 0.73 & 4.50 & 0.066 & $?$ & $?$ & $?$ & 0.566 \\
9532219 & 16.118 & 55001.945246 & 0.198155 & 5031 & -0.539 & 1.06 & 4.38 & 0.159 & $?$ & $?$ & $?$ & $?$ \\
\hline
\end{tabular}
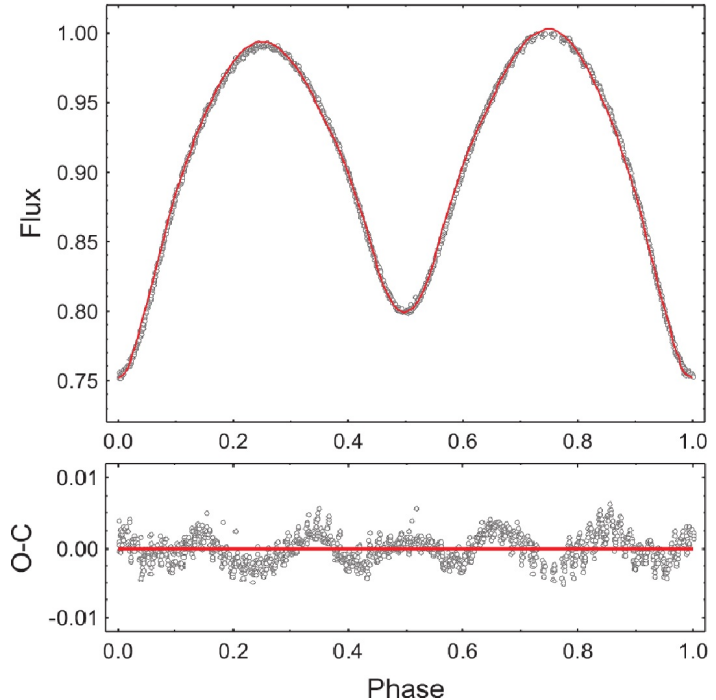

Fig. 3 The light curve of KID 8108785 and our fit (top) and the corresponding residuals (bottom)

Initially the primary temperature was fixed as $T_{1}=T_{m}$. The mean target temperatures $T_{m}$ (Table 1 ) have been estimated using dedicated pre-launch ground-based optical multi-color photometry plus Two Micron All Sky Survey (2MASS) $J, K$, and $H$ magnitudes (Skrutskie et al., 2006).

We varied the secondary temperature $T_{2}$, mass ratio $q$, orbital inclination $i$ and potentials $\Omega_{1,2}$ (and simultaneously relative radii $r_{1,2}$ and fillout factors $F F_{1,2}$ ). In order to reproduce the O'Connell effect we added cool spots on the primary and varied their parameters: longitude $\beta$, latitude $\lambda$, angular size $\alpha$ and temperature factor $k=T_{s p} / T_{1}$.

We adopted coefficients of gravity brightening 0.32 and reflection effect 0.5 appropriate for late stars. The limbdarkening coefficients were chosen according to the tables of Van Hamme (1993).

Finally, we varied the component temperatures around $T_{m}$ to search for the best fit.

The derived parameters of the targets corresponding to our light curve solutions are given in Table 2 while Table 3 presents information about the spot parameters. The formal PHOEBE errors of the fitted parameters are quite small (Tables 2-3) due to the high precision of the Kepler data.

It should be noted that the ephemerides from the Kepler EB catalogue (PRSA11) did not lead to the best fits. For this aim we varied the parameter "shift" of PHOEBE which final values are given in column 2 of Table 2.
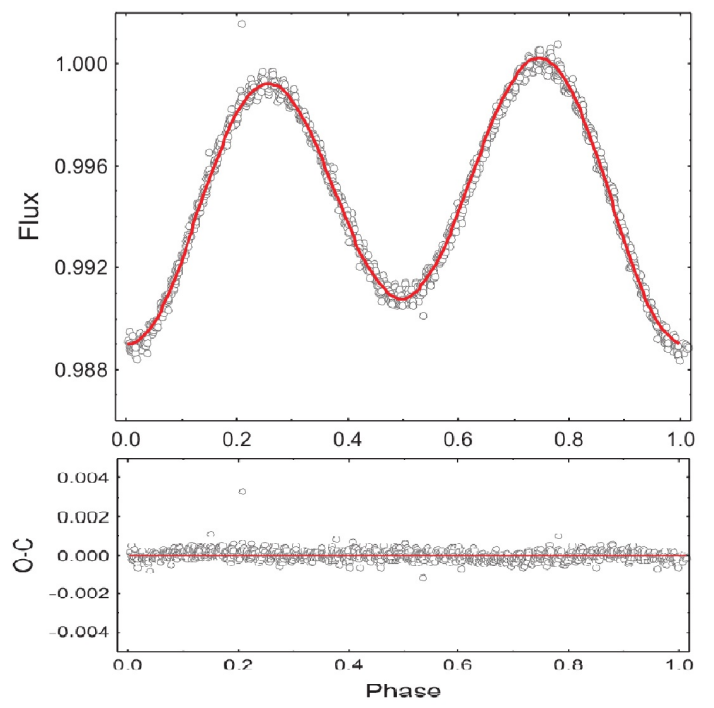

Fig. 4 The light curve of KID 6309193 and our fit (top) and the corresponding residuals (bottom)
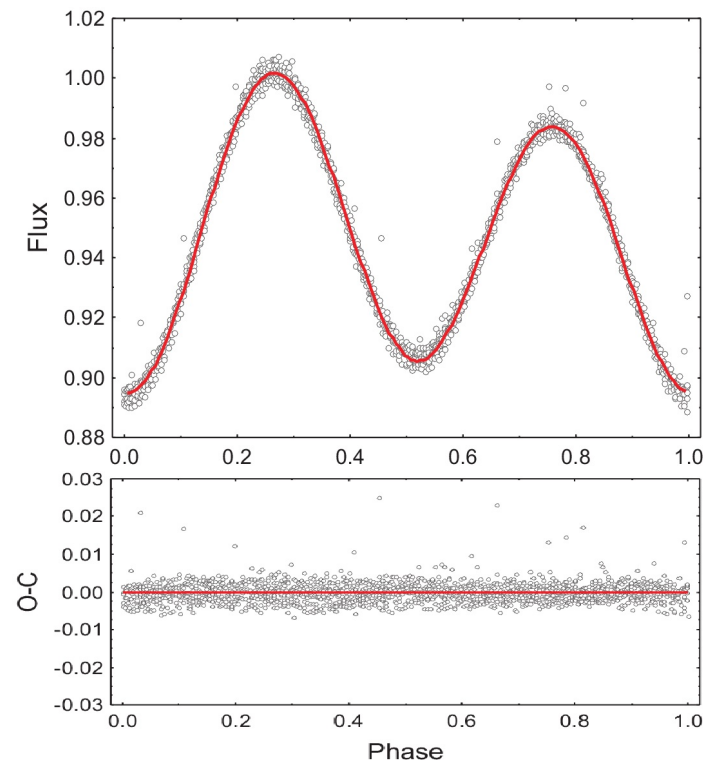

Fig. 5 The light curve of KID 12055255 and our fit (top) and the corresponding residuals (bottom) 
Table 2 Parameters of the best light curve solutions

\begin{tabular}{|c|c|c|c|c|c|c|c|c|c|c|c|}
\hline KID & shift & $\begin{array}{c}i \\
{\left[{ }^{\circ}\right]}\end{array}$ & $q$ & $\begin{array}{l}T_{1,2} \\
{[\mathrm{~K}]}\end{array}$ & $T_{2} / T_{1}$ & $\Omega_{1,2}$ & $\mathrm{FF}_{1,2}$ & $r_{1,2}^{\text {mean }}$ & $l_{1}$ & config & variability \\
\hline 4921906 & 0.0126 & $\begin{array}{c}21.04 \pm 0.02 \\
(26,4)\end{array}$ & $\begin{array}{c}0.5977 \pm 0.0005 \\
(0,47)\end{array}$ & $\begin{array}{l}4528 \pm 14 \\
4348 \pm 12\end{array}$ & $\begin{array}{l}0.960 \\
(0.958)\end{array}$ & $\begin{array}{l}3.0634 \pm 0.0002 \\
3.0634 \pm 0.0002\end{array}$ & $\begin{array}{l}0.0 \\
0.0\end{array}$ & $\begin{array}{l}0.424 \\
0.334\end{array}$ & 0.66 & $\begin{array}{c}\mathrm{C} \\
(\mathrm{OC})\end{array}$ & ellipsoidal \\
\hline 1572353 & -0.01923 & $\begin{array}{c}43.84 \pm 0.02 \\
\text { (33) }\end{array}$ & $\begin{array}{c}0.9577 \pm 0.0003 \\
(0.29)\end{array}$ & $\begin{array}{l}4525 \pm 20 \\
4277 \pm 13\end{array}$ & $\begin{array}{r}0.945 \\
(0.883)\end{array}$ & $\begin{array}{c}3.688 \pm 0.0003 \\
3.688 \pm 0.001\end{array}$ & $\begin{array}{l}0.0 \\
0.0\end{array}$ & $\begin{array}{l}0.383 \\
0.374\end{array}$ & 0.58 & $\begin{array}{c}\mathrm{C} \\
\text { (OC) }\end{array}$ & $\begin{array}{l}\text { grazing } \\
\text { eclipses }\end{array}$ \\
\hline 8108785 & 0.00147 & $\begin{array}{c}61.78 \pm 0.02 \\
(69.9)\end{array}$ & $\begin{array}{c}0.8309 \pm 0.0003 \\
(0.33)\end{array}$ & $\begin{array}{l}4711 \pm 4 \\
4246 \pm 3\end{array}$ & $\begin{array}{l}0.901 \\
(0.855)\end{array}$ & $\begin{array}{l}3.4664 \pm 0.0004 \\
3.4664 \pm 0.0006\end{array}$ & $\begin{array}{l}0.0 \\
0.0\end{array}$ & $\begin{array}{l}0.395 \\
0.360\end{array}$ & 0.62 & $\begin{array}{c}\mathrm{C} \\
(\mathrm{OC})\end{array}$ & eclipses \\
\hline 6309193 & -0.0196 & $\begin{array}{c}13.445 \pm 0.014 \\
\text { (21) }\end{array}$ & $\begin{array}{c}0.8007 \pm 0.0003 \\
(0.60)\end{array}$ & $\begin{array}{c}5435 \pm 2.5 \\
4751 \pm 2\end{array}$ & $\begin{array}{l}0.874 \\
(1.012)\end{array}$ & $\begin{array}{l}3.4165 \pm 0.0005 \\
3.4165 \pm 0.0005\end{array}$ & $\begin{array}{l}0.0 \\
0.0\end{array}$ & $\begin{array}{l}0.398 \\
0.359\end{array}$ & 0.68 & $\begin{array}{c}\mathrm{C} \\
(\mathrm{OC})\end{array}$ & ellipsoidal \\
\hline 12055255 & 0.0128 & $\begin{array}{c}39.79 \pm 0.05 \\
(?)\end{array}$ & $\begin{array}{c}0.9637 \pm 0.0005 \\
(?)\end{array}$ & $\begin{array}{c}3701 \pm 8 \\
3744 \pm 10\end{array}$ & $\begin{array}{l}1.011 \\
(?)\end{array}$ & $\begin{array}{l}3.6326 \pm 0.0022 \\
3.6936 \pm 0.0012\end{array}$ & $\begin{array}{l}0.128 \\
0.019\end{array}$ & $\begin{array}{l}0.392 \\
0.377\end{array}$ & 0.55 & (?) & $\begin{array}{l}\text { grazing } \\
\text { eclipses }\end{array}$ \\
\hline 9532219 & 0.00647 & $\begin{array}{c}68.42 \pm 0.06 \\
(?)\end{array}$ & $\begin{array}{c}0.9320 \pm 0.0022 \\
\text { (?) }\end{array}$ & $\begin{array}{l}4950 \pm 22 \\
4867 \pm 21\end{array}$ & $\begin{array}{c}0.983 \\
(?)\end{array}$ & $\begin{array}{l}3.5687 \pm 0.0022 \\
3.5873 \pm 0.0028\end{array}$ & $\begin{array}{l}0.172 \\
0.164\end{array}$ & $\begin{array}{l}0.398 \\
0.388\end{array}$ & 0.55 & $\begin{array}{l}\text { OC } \\
\text { (?) }\end{array}$ & eclipses \\
\hline
\end{tabular}

Note: $\mathrm{C}$ means contact binaries, OC - overcontact systems
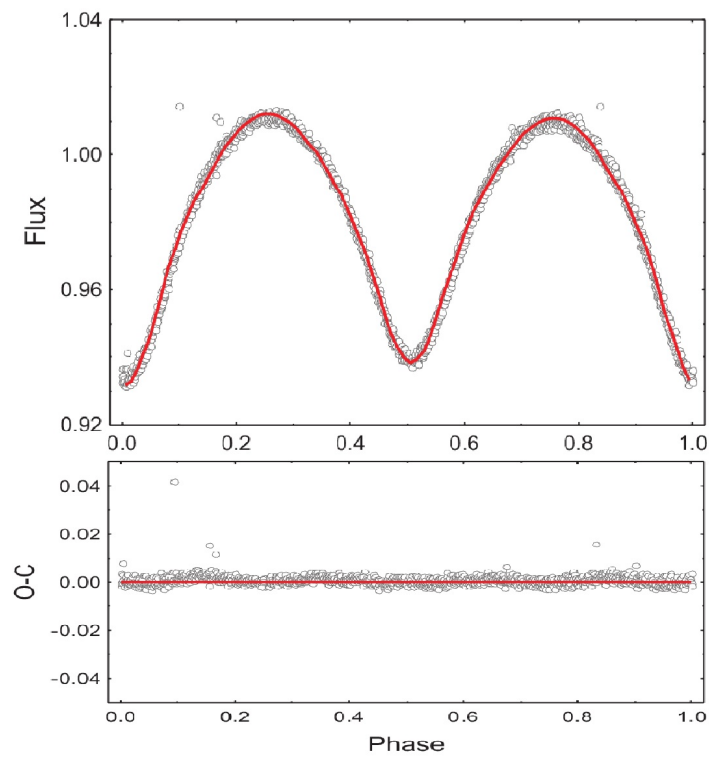

Fig. 6 The light curve of KID 9532219 and our fit (top) and the corresponding residuals (bottom)

Table 3 Parameters of the spots and third light of the targets

\begin{tabular}{rccccc}
\hline KID & $\begin{array}{c}\beta \\
\left.{ }^{\circ}\right]\end{array}$ & $\begin{array}{c}\lambda \\
{\left[{ }^{\circ}\right]}\end{array}$ & $\begin{array}{c}\alpha \\
{\left[{ }^{\circ}\right]}\end{array}$ & $k$ & $l_{3}$ \\
& $75.0 \pm 0.1$ & $88.1 \pm 0.1$ & $11.0 \pm 0.1$ & $0.901 \pm 0.001$ & 0 \\
\hline 4921906 & $91.5 \pm 0.1$ & $329.9 \pm 0.1$ & $17.6 \pm 0.1$ & $0.900 \pm 0.002$ & 0 \\
1572353 & $126.3 \pm 0.2$ & $279.6 \pm 0.3$ & $24.9 \pm 0.2$ & $0.939 \pm 0.001$ & 0 \\
8108785 & $63.9 \pm 0.1$ & $338.4 \pm 0.1$ & $10.3 \pm 0.2$ & $0.940 \pm 0.001$ & 0 \\
6309193 & $89.9 \pm 0.2$ & $14.9 \pm 0.2$ & $0.900 \pm 0.001$ & 0 \\
12055255 & $59.6 \pm 0.2$ & $89.9 \pm \pm 0.04$ & $10.1 \pm 0.7$ & $0.914 \pm 0.007$ & 0.78 \\
9532219 & $81.5 \pm 0.6$ & $70.09 \pm 0.04$ & & & \\
\hline
\end{tabular}

The synthetic curves corresponding to the parameters of our light curve solutions are shown in Figs. 1-6 as red continuous lines.

The derived photometric mass ratio requires a special attention. Usually this parameter needs spectral observations. But the spectral mass ratio for short-period binaries with fast rotation is quite imprecise due to the line blending while the geometrical effects and mass ratio are much more important in defining the shapes of their light curves and the eclipse depths. Hence, the values of the photometric mass ratio for ultrashort-period Kepler binaries should be considered with high confidence.

There are differences between our solutions and those obtained by automated fitting cited in the Kepler EB catalog (the values in parentheses in Table 2).

(1) The most orbital inclinations in PRSA11 are bigger than ours.

(2) The mass ratios in PRSA11 are considerably smaller than our values (Table 2 ).

(3) We had to assume a third light $l_{3}$ only for KID 9532219 (Table 3) while the values of this parameter in PRSA11 were nonzeros for the all six targets. We found a weaker star close to KID 9532219 (at distance around 6 arcsec) that could explain the presence of $l_{3}$ in the light curve solution of this target.

(4) There are no values of the potentials $\Omega_{1,2}$ (and thus of the relative radii $r_{1,2}$ and fillout factors $\left.F F_{1,2}\right)$ as well as of relative luminosities in PRSA11 that means that the automated modelling leads to approximate results.

There are several explanations for the differences between our "manual" solutions and those of the automated fitting.

(a) The EBAI method fixes $l_{3}=1$ - crowding while this parameter is invoked in our procedure only when a satisfactory fit is impossible for any combination of the parameters. The bigger value of $l_{3}$ requires bigger light amplitude and correspondingly bigger orbital inclination. Moreover, due to the correlation between third light and mass ratio for partially eclipsing binaries the bigger third light leads to the larger mass ratio for fixed inclination angle.

(b) Although all light curves exhibit O'Connell effect (Figs. 1-6) the EBAI method does not take into account the effect from spots. Then the results of the automated modelling should be assumed as first approximations of the fitted parameters. 


\section{Analysis of the results}

The analysis of our light curve solutions of the ultrashortperiod binaries from the Kepler data base led to several conclusions.

(1) The configurations of all targets are almost the same: 4 of them are contact binaries (KID 4921906, KID 1572353, KID 8108785, KID 6309193) and 2 are slightly overcontact systems (KID 12055255 and KID 9532219).

(2) Two targets, KID 4921906 and KID 6309193, are noneclipsing systems. The registration of their lowamplitude ellipsoidal and rotational (due spots) variability is possible due to the high Kepler accuracy. There are quite many objects of this type in the Kepler EB catalog and hence, it should be called catalog of photometrically-double stars.

(3) To model the data of KID 9532219 we had to assume considerable contribution of a third light $l_{3}$. It originates probably from the close neighbor. We do not exclude this fainter star to be the true variable because the size of the Kepler pixels projected onto the sky (4 arcsec) coupled with the high star density near the Galactic plane lead to a non-negligible likelihood of associating an EB event with the wrong star (PRSA11).

(4) The target KID 12055255 is very rare case of ultrashortperiod binary consisting of two M dwarfs. The system seems to be overcontact. We are not aware of any overcontact binary consisting of $\mathrm{M}$ dwarfs.

(5) Feldmeier et al. (2011) classified the target KID 9532219 as a $\delta$ Sct variable (from their groundbased photometric observations of the Kepler FoV for the pre-launch survey). The Kepler data and our modeling certainly reveal that this target (or its neighbor) is eclipsing binary.

(6) Recently KID 6309193 was noted as "false" in the Kepler EB catalog. Our modeling showed that it is not eclipsing star but its light curve can be reproduced by ellipsoidal variations of binary. However we do not exclude the alternative the observed variability to be inherent to some of its two considerably fainter neighbors (at distances around 4.5 arcsec and 7 arcsec).

(7) The ripples in the residuals of KID 8108785 (Fig. 3) seem to be quasi-sinusoidal with period of $55 \mathrm{~min}$ and amplitude of $0.005 \mathrm{mag}$. Although this variability is visible during all phases, it could be explained by inherent changes of some of the components of this system due to its small orbital inclination.

Although all considered light curves reveal O'Connell effect and are reproduced by cool spots we have not studied long-term light curve changes as a result of the evolution of these surface inhomogeneities. To do this one should have detailed information about the problems of the satellite steering which introduce different trends into the data.

\section{Conclusions}

Our investigation added six new members to the small family of the studied ultrashort-period binaries with MS components.

All six targets turned out to be contact or slightly overcontact configurations which components are almost in thermal contact. Two targets, KID 4921906 and KID 6309193, are not eclipsing but reveal ellipsoidal and spot variability. KID 12055255 is very rare case of ultrashort-period binary consisting of two $\mathrm{M}$ dwarfs. We established that KID 9532219 is an eclipsing binary but not a $\delta$ Sct variable as it was previously supposed. One of the components of KID 8108785 exhibits inherent, quasi-sinusoidal, smallamplitude variability.

The analysis of the results showed that reliable global parameters of the Kepler binaries might be obtained only by standard method of light curve solutions. The automated EBAI method models the Kepler data quite roughly. Obviously, the numerous and exclusive precise Kepler data deserve precise light curve solutions by the standard way.

Acknowledgements. We used the live version of the Kepler EB catalog from http://keplerEBs.villanova.edu The research was supported partly by funds of project RD 08-244 of Scientific Foundation od Shumen University. This publication makes use of data products from the Two Micron All Sky Survey, which is a joint project of the University of Massachusetts and the Infrared Processing and Analysis Center/California Institute of Technology, funded by the National Aeronautics and Space Administration and the National Science Foundation. This research also has made use of the USNOFS Image and Catalogue Archive operated by the United States Naval Observatory, Flagstaff Station (http://www.nofs.navy.mil/data/fchpix/).

The authors are grateful to anonymous referee for the valuable notes and propositions.

\section{References}

Dimitrov D., Kjurkchieva D., 2010, MNRAS 406, 2559

Dimitrov D., Kjurkchieva D., Radeva V., 2012, BlgAJ 18c, 81

Duerbeck H., 1997, IBVS No4513

Feldmeier J. et al., 2011, AJ 142, 2

Lohr, M. E.; Hodgkin, S. T.; Norton, A. J.; Kolb, U. C., 2014, A \& A 563A, 34

Maceroni C., Montalban J., 2004, A \& A 426, 577

Maceroni C., Rucinski S.M., 1997, PASP 109, 782

Matuevic G. et al., 2012, AJ 143, 123

Pribulla T., Vanko M., Hambalek L., 2009, IBVS No.5886

Prsa A., Zwitter T., 2005, ApJ 628, 426

Prsa A. et al., 2008, ApJ 687, 542

Prsa A. et al., 2011, AJ 141, 83

Qian S.-B. et al., 2014, CoSka 43, 290

Pribulla T. et al., 2009, AJ 137, 3646

Rucinski S., Duerbeck H., 1997, PASP 109, 1340

Rucinski S., 1992, AJ 103, 960

Rucinski S., 2006, MNRAS 368, 319

Rucinski S., 2007, MNRAS 382, 393

Skrutskie M.F. et al., 2006, AJ, 131, 1163

Slawson R. et al., 2011, AJ 142, 160

Southworth J. et al., 2011, MNRAS 414, 3740 
Steffen J. et al., 2011, MNRAS 417L, 31

Vandenberg D., Clem J., 2003, AJ 126, 778

Van Hamme W., 1993, AJ 106, 2096

Weldrake D., Sackett P., Bridges T., Freeman K., 2004, AJ 128, 736

Welsh W. et al., 2011, ApJS 197, 4

Winn J. et al., 2011, ApJ 741L, 1 